\title{
Some consequences of exchangeability in random-matrix theory
}

\author{
G. Le Caër ${ }^{1, *}$ and R. Delannay ${ }^{2, \dagger}$ \\ ${ }^{1}$ Division of Engineering and Applied Science, Mail Stop 138-78, California Institute of Technology, Pasadena, California 91125 \\ ${ }^{2}$ Groupe Matière Condensée et Matériaux, CNRS UMR C6626, Université de Rennes-I, Avenue du Général Leclerc, \\ F-35042 Rennes Cedex, France \\ (Received 14 August 1998)
}

\begin{abstract}
Properties of infinite sequences of exchangeable random variables result directly in explicit expressions for calculating asymptotic densities of eigenvalues $\rho_{\infty}(\lambda)$ of any ensemble of random matrices $H$ whose distribution depends only on $\operatorname{tr}\left(H^{\dagger} H\right)$, where $H^{\dagger}$ is the Hermitian conjugate of $H$. For real symmetric matrices and for Hermitian matrices, the densities $\rho_{\infty}(\lambda)$ are constructed by summing up Wigner semicircles with varying radii and weights as confirmed by Monte Carlo simulations. Extensions to more general matrix ensembles are also considered. [S1063-651X(99)09504-5]
\end{abstract}

PACS number(s): 02.50.- r, 05.45.-a, 21.10. $-\mathrm{k}, 72.80 . \mathrm{Ng}$

The "explosive" development of random-matrix theory (RMT) during the last decade has been covered recently by Guhr et al. [1], who emphasize the universal applicability of RMT to the fluctuation properties on local scales defined by mean level spacings of quantum (and of certain classical) systems. Indeed, RMT is used considerably in various branches of physics, notably in nuclear physics, quantum chaology, and for investigating Hamiltonians of disordered and strongly interacting quantum systems ([1-7] and references therein). Properties of distributions of eigenvalues, and to a lesser extent of eigenvectors, of large random matrices have been thoroughly investigated for various specific and general random-matrix ensembles (RME). Among the three fundamental Gaussian ensembles (GE) [1,2], we mention the two associated with real symmetric (GOE) and with Hermitian (GUE) $N \times N$ random matrices $H_{N}$. They are named after the orthogonal and unitary transformations that leave the corresponding ensemble statistically invariant. The number of distinct real random variables that are necessary to construct each $N \times N$ matrix is $N_{p}$, with $N_{p}=N+\beta N_{m}, N_{m}$ $=N(N-1) / 2$ and $\beta=1,2$ for the GOE and GUE respectively $[1,2]$. A random variable whose distribution is Gaussian with a zero mean and a variance $\sigma^{2}$ is hereafter denoted as $N\left(0, \sigma^{2}\right)$. The previous $N_{p}$ variables that constitute the matrix elements $H_{i j}$ are independent $N\left[0, \sigma^{2}\left(1+\delta_{i j}\right) / 2\right]$ variables. The GOE and GUE probability density function is then $G_{\beta, \sigma}\left(H_{N}\right)=K_{\beta, N} \exp \left[-\operatorname{tr}\left(H_{N}^{2}\right) /\left(2 \sigma^{2}\right)\right]$, where tr means trace. The variance is taken as $\sigma^{2}=\alpha_{G}^{2} / N, \alpha_{G}=$ const, to obtain a spectrum independent of $N$ when $N \rightarrow \infty$. In contrast to the universality of GE local fluctuations around mean values $[1,2]$, their asymptotic average density of eigenvalues $\rho_{W}(\lambda)$ [Eq. (1)] is a bound distribution whose shape is unrealistic for most physical systems [1]. As the GE's are ergodic, the empirical distribution function $F_{N}(x)$ $=($ number of $\lambda \leqslant x) / N$ of every large matrix tends also to a

\footnotetext{
*Permanent address: LSG2M, CNRS UMR 7584, Ecole des Mines, F-54042 Nancy Cedex, France.

Electronic address: lecaer@mines.u-nancy.fr

${ }^{\dagger}$ Electronic address: Renaud.Delannay@univ-rennes1.fr
}

distribution function whose associated probability density is $\rho_{W}(\lambda)$, the classical Wigner semicircle [1-3] $\left(a^{2}=2 \beta \alpha_{G}^{2}\right.$, $\Lambda=|\lambda-c|)$ :

$$
\rho_{W}(\lambda)=\left(2 / \pi a^{2}\right)\left(a^{2}-\Lambda^{2}\right)^{1 / 2} \text { for } \Lambda \leqslant a
$$

(and 0 for $\Lambda>a$ ) whose scale parameter is its "radius" $a$ and whose centering parameter is $c\left(c=\left\langle\operatorname{tr} H_{N}\right\rangle / N=0\right.$ for the GOE and GUE). However, recent sophisticated developments, particularly on noncommutative probability theory, have created significant new results on the algebra of random matrices and have shed light on the theoretical importance of the Wigner semicircular distribution [4-7]. Its further significance as a reference density for constructing large- $N$ densities $\rho_{\infty}(\lambda)$, bound or not, of broad RME classes is discussed in the present paper.

The set $M_{N}(\mathrm{R})$ [or $\left.M_{N}(C)\right]$ of matrices with real (or complex) entries is an $N \times N$ (or $2 N \times 2 N$ )-dimensional vector space over the field of real numbers $\mathrm{R}$. The norm of a matrix $H_{N}$ is taken here as $\left\|H_{N}\right\|=\left[\operatorname{tr}\left(H_{N}^{\dagger} H_{N}\right)\right]^{1 / 2}$, where $H_{N}^{\dagger}$ is the Hermitian conjugate of $H_{N}$. It gives to $M_{N}(\mathrm{R})$ [or $\left.M_{N}(C)\right]$ a structure of Euclidean space. Any $d$-dimensional subspace $S_{d}$ of $M_{N}(\mathbb{R})$ or $M_{N}(C)$, given a basis, is isomorphic to the space $\mathbb{R}^{d}$. Each matrix $H_{N}$ of $S_{d}$ is thus in a one-to-one correspondence with a vector $\mathbf{X}$ of $\mathbb{R}^{d}$, denoted here $\mathbf{X}=\mathcal{V}\left(H_{N}\right)$. The inverse transformation from $\mathbf{X}$ to $H_{N}$ is denoted as $H_{N}=\mathcal{M}(\mathbf{X})$. If the basis of $S_{d}$ is orthonormal, the usual Euclidean norm of $\mathbf{X}$ is equal to $\left\|H_{N}\right\|$. The dimension $d=N_{p}$ is imposed by the symmetry of the RME, which is put in isometric correspondence with $\mathbb{R}^{d}$. We define $R_{i j}$ (or $C_{i j}$ ) to be the matrix having a lone 1 (or $\sqrt{-1}$ ) as $(i, j)$ entry and all the others equal to 0 . The family of $R_{i j}$ (or $R_{i j}$ and $\left.C_{i j}\right)(1 \leqslant i, j \leqslant N)$ is a canonical orthonormal basis of $M_{N}(\mathbb{R}) \quad\left[\right.$ or $\left.\quad M_{N}(C)\right]$. The family $\quad\left[\left\{R_{i j}\right\},\left\{\left(R_{i j}\right.\right.\right.$ $\left.\left.\left.+R_{j i}\right) / \sqrt{2}\right\},\left\{\left(C_{i j}-C_{j i}\right) / \sqrt{2}\right\}\right](1 \leqslant i<j \leqslant N)$ is, for example, an orthonormal basis of the $N_{p}=N^{2}$-dimensional subspace of Hermitian matrices, and hence the associated $\mathbf{X}$ components are $\left\{H_{i i}\right\},\left\{\sqrt{2} \operatorname{Re}\left(H_{i j}\right)\right\},\left\{\sqrt{2} \operatorname{Im}\left(H_{i j}\right)\right\},(1 \leqslant i<j \leqslant N)$. The basis reduces to $\left\{\left(C_{i j}-C_{j i}\right) / \sqrt{2}\right](1 \leqslant i<j \leqslant N)\left[N_{p}=N(N\right.$ $-1) / 2]$ if the matrices are further antisymmetric. A description of a stacking order is unnecessary here, as we consider 
only RME's associated with random vectors $\mathbf{X}$ whose components are $N_{p}$ exchangeable [8].

The distribution function of $\mathbf{X}$ is said to be $N_{p}$ exchangeable if it remains unchanged by any permutation of the $\mathbf{X}$ components. A $k$-exchangeable sequence $\left(X_{1}, \ldots, X_{k}\right)$ is $n$ extendible $(n>k)$ if it is such that $\left(X_{1}, \ldots, X_{k}\right)$ $\stackrel{d}{=}\left(X_{1}^{\prime}, \ldots, X_{k}^{\prime}\right)$ for some $n$-exchangeable sequence $\left(X_{1}^{\prime}, \ldots, X_{n}^{\prime}\right)$, where $\stackrel{d}{=}$ means that both members have the same distribution. An infinite sequence of random variables $\left\{X_{k}\right\}, k=1, \ldots, \infty$, is exchangeable if it is $n$ exchangeable for every finite $n$. It is a mixture of identically and independently distributed (IID) sequences as demonstrated by the de Finetti's theorem, which is valid in particular in Euclidean spaces [8-14]. Then, there exists a $p$-dimensional $(p \geqslant 1)$ random variable $\mathbf{Y}$ such that the components of $\mathbf{X}=\left(X_{1}, \ldots, X_{n}\right)$ are conditionally IID random variables; that is, $\operatorname{Prob}\left(X_{1}\right.$ $\left.\leqslant x_{1}, \ldots, X_{n} \leqslant x_{n}\right)=\prod_{i=1}^{n} \operatorname{Prob}\left(X_{i} \leqslant x_{i} \mid \mathbf{Y}=\mathbf{y}\right), \quad$ given $\quad \mathbf{Y}=\mathbf{y}$ [11]. Finite versions of the de Finetti theorem further show how $n$-exchangeable sequences are close, in terms of a distance between distributions, to mixtures of IID sequences $[8,9]$. For instance, if a $k$-exchangeable sequence is $n$-extendible with $n$ large as compared to $k^{2}$, the former sequence is close to a mixture of IID sequences [8]. We discuss below some notable RME's for which the $N_{p}$-exchangeable sequences of $\mathbf{X}$ components yield, when $N_{p} \rightarrow \infty$, infinite exchangeable sequences which are mixtures of known "reference" sequences $\mathbf{R}$. The properties of some permutationinvariant distributions are more naturally expressed in terms of characteristic functions (c.f.). The distribution of $\mathbf{X}$ is, for example, called $\alpha$ symmetric $(0<\alpha \leqslant 2)[10,12]$ and references therein] or spherical for $\alpha=2$ [11-14] if its c.f. is

$$
\phi(\mathbf{t})=\langle\exp (i \mathbf{t} \cdot \mathbf{X})\rangle=\phi\left[t=\left(\sum_{k=1}^{N_{p}}\left|t_{k}\right|^{\alpha}\right)^{1 / \alpha}\right]
$$

for all real $N_{p}$-dimensional vectors $\mathbf{t}$. We denote by $F_{N_{p}}(\alpha)$ the class of such c.f. For $\alpha \neq 2$, the representation of functions whose c.f. are given by Eq. (2) is solely known for $\alpha$ $=1[10,12]$. For the spherical case, the density of $\mathbf{X}$ depends only on $\|\mathbf{X}\|$ whatever $N_{p}$, as its c.f. depends only on the modulus $t$ of $\mathbf{t}$ and conversely [13]. The density of $H_{N}$ $=\mathcal{M}(\mathbf{X})$ if it exists is consequently $f\left(\operatorname{tr}\left(H_{N}^{\dagger} H_{N}\right)\right)$. By extension, the latter RME's will be named "spherical" hereafter. For the spherical case, $\phi(t)$ is given by Eq. (2) if and only if it is represented by $[12,13]$

$$
\phi(t)=\int_{0}^{\infty} \Omega_{N_{p}}(r t) d P_{N_{p}}(r)
$$

for some distribution $P_{N p}(r)$ which is assumed to have no mass at zero, where $\Omega_{N_{p}}(t)$ is the c.f. of a vector $\mathbf{U}$ which is uniformly distributed on the surface of the unit sphere in $\mathrm{R}^{N_{p}}$ $[12,13], \Omega_{N_{p}}(t)=\Gamma\left(N_{p} / 2\right)(2 / t)^{m} J_{m}(t), m=\left(N_{p}-2\right) / 2$ and $J_{m}$ is a Bessel function. An expression similar to Eq. (3) which involves a c.f. different from $\Omega_{N_{p}}(t)$ is also known for $\alpha=1$ [12]. As proven by Bretagnolle et al. (see [12]), Eq. (2) admits the representation

$$
\phi(t)=\int_{0}^{\infty} \exp \left(-r t^{\alpha}\right) d F(r)
$$

for $\phi(t)$ belonging to the class $F_{\infty}(\alpha)$. Equation (4) yields the asymptotic distribution of $\alpha$-symmetric $\mathbf{X}$ as a mixture, defined by the distribution $F(r)$, of reference vectors $\mathbf{R}$ whose c.f. is $\exp \left(-r t^{\alpha}\right)$. The components of $\mathbf{R}$ are then IID according to a Lévy stable distribution, which is $N(0,2 r)$ for $\alpha=2[8,10-14]$. The distribution of a finite $k$-exchangeable spherical sequence which is $n$ extendible $(n \gg k)$ is also well approximated by a mixture of normal distributions [8,9]. From the distributions of $\mathbf{X}$ and $\mathbf{R}$, we deduce that any $\alpha$-symmetric RME is asymptotically a mixture of random Lévy matrices of the same symmetry with independent distinct entries (see [15] for a study of real-symmetric Lévy RME's). In particular, any spherical RME is asymptotically a mixture of Gaussian ensembles with the same symmetry. Such spherical RME's are thus not ergodic, as every large- $N$ extendible random matrix $H_{N}$ is a Gaussian matrix. The universality of the spectral fluctuations of such $H_{N}$ 's is thus a straightforward consequence of that of the reference GE's. For orthogonal ( $\beta=1$, real-symmetric matrices) or unitary ( $\beta=2$, Hermitian matrices) extendible spherical RME's, the density of $H_{N}$ which results from Eq. (4) for large $N$ is thus written as

$$
P_{\beta}\left(H_{N}\right)=K_{\beta, N} f\left[\operatorname{tr}\left(H_{N}^{2}\right)\right]=\int_{0}^{\infty} G_{\beta, \sigma}\left(H_{N}\right) d W(\sigma)
$$

for some distribution $W(\sigma)$. The elements of $H_{N}$ are then asymptotically independent under the prior condition that $\sigma$ has a given value $\sigma_{0}$. The reference GE's are the only spherical RME's with independent matrix elements.

For orthogonal and unitary spherical RME's, extendible or not, we deduce from Eqs. (3) and (4) that the ensembleaveraged asymptotic density is $\rho_{\infty}(\lambda)=\lim _{N \rightarrow \infty}\left[\rho_{N}(\lambda)\right]$, where $\rho_{N}(\lambda)$ is a mixture of $\rho_{\text {unif }}(\lambda)$, the eigenvalue density of a "uniform" orthogonal or unitary RME $U_{N}=\mathcal{M}(\mathbf{U})$ [see below Eq. (3)], for which $\operatorname{tr}\left(U_{N}^{2}\right)$ is constant. The asymptotic eigenvalue density $\rho_{\text {unif }}(\lambda)$ is indeed $\rho_{W}(\lambda)$ from numerical simulations and exact calculations [16]. The latter indicate that $\rho_{\text {unif }}(\lambda)$ is actually very well approximated by $\rho_{W}(\lambda)$ for $N$ as small as 50. For large $N$, the GOE and GUE tend in fact to fixed-trace ensembles as $z=\operatorname{tr}\left(H_{N}^{2}\right) / \sigma^{2}$ has a $\chi$-square distribution with $N_{p}$ degrees of freedom which results in $\langle(z$ $\left.-\langle z\rangle)^{2}\right\rangle^{1 / 2} /\langle z\rangle \alpha N^{-1}$. The asymptotic density is finally

$$
\rho_{\infty}(\lambda)=\lim _{N \rightarrow \infty} \int_{|\lambda|}^{\infty} \gamma_{N} r^{\left(N_{p}-3\right)} f\left(N r^{2} / 4\right)\left(r^{2}-\lambda^{2}\right)^{1 / 2} d r,
$$

with

$$
\varphi_{N}=\int_{0}^{\infty} r^{\left(N_{p}-1\right)} f\left(r^{2}\right) d r, \quad \gamma_{N}=N^{\left(N_{p} / 2\right)} /\left(2^{\left(N_{p}-1\right)} \pi \varphi_{N}\right),
$$

where $f\left(\operatorname{tr}\left(H_{N}^{\dagger} H_{N}\right)\right)$ is the density defined in Eq. (5). More generally, the asymptotic density [Eq. (6)] can be rewritten as 


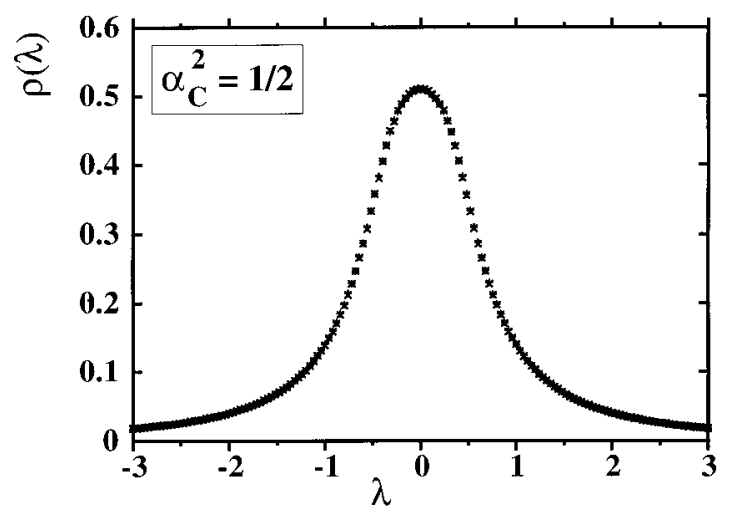

FIG. 1. Comparison of the binned distributions $\rho(\lambda)$ $=($ average number of eigenvalues in $[\lambda-\Delta \lambda / 2, \lambda+\Delta \lambda / 2]) /(N \Delta \lambda)$ with the binned theoretical distribution calculated from Eq. (9) (-) for the CLOE; $\rho(\lambda)$ is calculated from Monte Carlo simulations with $\Delta \lambda=0.04,4 \times 10^{5}$ matrices and $N=50(x), N=100(+)$.

$$
\rho_{\infty}(\lambda)=2 \pi^{-1} \int_{\lambda^{2}}^{\infty}\left(u-\lambda^{2}\right)^{1 / 2} u^{-1} d F(u)
$$

for all spherical RME's whose reference density is a Wigner semicircle. Calculation of $\rho_{\infty}(\lambda)$ for spherical ensembles of all kinds of symmetries either with real or with complex eigenvalues might similarly be performed from Eq. (4) [16]. We describe below the example of orthogonal (CLOE) and of unitary (CLUE) Cauchy-Lorentz ensembles, whose associated $\mathcal{V}\left(H_{N}\right)$ are distributed according to a spherical Cauchy distribution [10], with a c.f. $\phi(\mathbf{t})=\exp$ $\left(-\gamma\left[\sum_{k=1}^{N_{p}} t_{k}^{2}\right]^{1 / 2}\right)$ :

$$
P_{\beta}\left(H_{N}\right)=\frac{K_{\beta, N}}{\left[1+\operatorname{tr}\left(H_{N}^{2}\right) / \gamma^{2}\right]^{\left(N_{p}+1\right) / 2}} .
$$

With $\gamma^{2}=\alpha_{C}^{2} / N,\left(\alpha_{C}=\right.$ const $)$, Eq. (6) yields

$$
\rho_{\beta, \infty}(\lambda)=C\left[1-\int_{0}^{\pi / 2} \cos (\theta) \exp \left(-\beta \alpha_{C}^{2} \cos ^{2}(\theta) / \lambda^{2}\right) d \theta\right],
$$

with $C=2 \pi^{-3 / 2} \beta^{-1 / 2} \alpha_{C}^{-1}$. For small $\lambda$, distribution (9) has a parabolic variation and is flatter than a Lorentz line whose maximum density is chosen as $C$. It decreases as $\lambda^{-2}$ as $\lambda$ $\rightarrow \pm \infty$ and has diverging moments. The CLOE density behaves for small and large $\lambda$ in the same way as the density of the real-symmetric Lévy ensemble with independent Cauchy entries [15]. Asymptotic eigenvalue densities independent of $N$ are, however, reached for different $N$ dependences of the scaling factor which varies as $N^{-1 / 2}$ for the CLOE $[\gamma, \mathrm{Eq}$. (8)], as it does for the $\operatorname{GOE}\left[\sigma\right.$, Eq. (5)], and as $N^{-1}$ for the Lévy ensemble [15]. Monte Carlo simulations (Fig. 1) were performed for the CLOE from the stochastic representation, $H_{N} \stackrel{d}{=} r U_{N}$, where $1 / r$ is the absolute value of a normal variable with zero mean [10] which is independent of $U_{N}$ $=\mathcal{M}(\mathbf{U})$. The vector $\mathbf{U}$ is obtained from $\mathbf{U} \stackrel{d}{=} \mathbf{G} /\|\mathbf{G}\|$, where $\mathbf{G}$ is a Gaussian vector with IID components. Equation (9) is easily generalized to orthogonal or unitary Student's [10] RME's for which the exponent of the Cauchy distribution

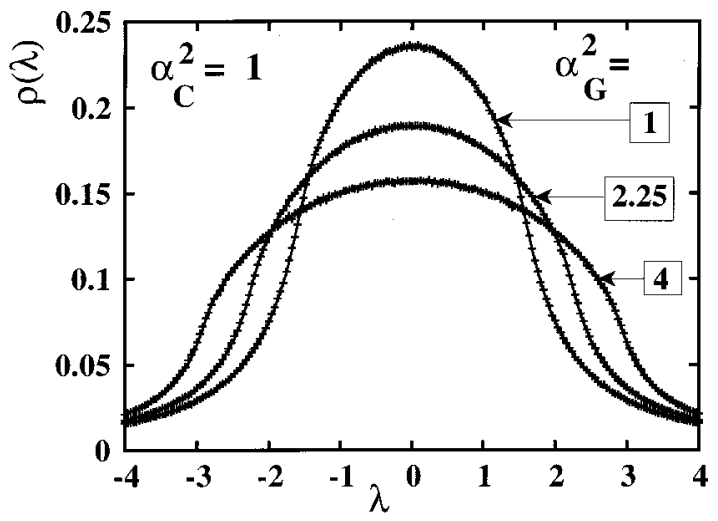

FIG. 2. Comparison of Monte Carlo distributions $\rho(\lambda)$ [see legend of Fig. $1, \Delta \lambda=0.04,2 \times 10^{5}$ matrices $\left.N=100(+)\right]$ with the binned theoretical distributions calculated from Eq. (10) (solid lines) for the sum of independent CLOE and GOE matrices.

$\left(N_{p}+1\right) / 2$ [Eq. (8)] is replaced by $\left(N_{p}+m\right) / 2$, where $m$ is an integer $(m>1)$. When $m$ increases, their asymptotic densities evolve progressively to the Wigner semicircle [16] as moments of order $k$ are finite for $k<m$ $\left(\rho_{\infty}(\lambda)\right.$ varies as $|\lambda|^{-(m+1)}$ when $\left.\lambda \rightarrow \pm \infty\right)$. Simulations [16] of the large- $N$ density of real eigenvalues of antisymmetric Hermitian Cauchy spherical ensembles confirm that $\rho_{\infty}(\lambda)$ is a sum of asymptotic eigenvalue densities of antisymmetric Hermitian GE with varying radii. The latter densities are modified Wigner semicircles that differ a little from $\rho_{W}(\lambda)$ in the central region [2]. The simulated radial density of complex eigenvalues agrees similarly with the theoretical density deduced from Eq. (4) for asymmetric Cauchy spherical matrices with complex elements $\left[N_{p}=2 N^{2}\right][16]$.

The large- $N$ eigenvalue distribution of the sum of independent matrices can be deduced from the law of addition for Blue's functions, the functional inverses of the Green's functions, for Hermitian RME's or of generalized Blue's functions for non-Hermitian RME's [6,7]. For independent spherical matrices of the same symmetry, it is simply shown, using c.f., that they are built from the sum of reference densities using the appropriate scale factors and weights. The large- $N$ density of the sum of two orthogonal or unitary spherical matrices $H_{N}=H_{1 N}+H_{2 N}$ is thus obtained from Eq. (7) where the distribution $F(u)$ of the square of the radius $u=u_{1}+u_{2}$ is calculated from the known distributions of the radius squares $u_{1}$ and $u_{2}$. The large- $N$ density of the sum of two independent matrices, one from the GOE with a degenerate distribution, $u_{1}=2 \alpha_{G}^{2}$ [see above Eq. (1)], and one from the CLOE with a density $f_{2}\left(u_{2}\right)=\alpha_{C} \pi^{-1 / 2} u_{2}^{-3 / 2} \exp$ $\left(-\alpha_{C}^{2} / u_{2}\right)\left(u_{2}>0\right)$ is, for example,

$$
\begin{aligned}
\rho(\lambda)= & C \int_{\max \left(\lambda^{2}, 2 \alpha_{G}^{2}\right)}^{\infty} S(u)^{-3 / 2}\left(u-\lambda^{2}\right)^{1 / 2} \\
& \times \exp \left[-\alpha_{C}^{2} / S(u)\right] u^{-1} d u,
\end{aligned}
$$

where $C=2 \alpha_{C} \pi^{-3 / 2}, S(u)=u-2 \alpha_{G}^{2}$. For computer calculations of Eq. (10) (Fig. 2), we use the fact that $S^{-1 / 2}$ is distributed as the absolute value of $N\left[0,1 /\left(2 \alpha_{C}^{2}\right)\right]$.

Equations (6) and (7) and their generalizations may appear as intuitively obvious for RME's whose density is 
$f\left(\operatorname{tr}\left(H_{N}^{\dagger} H_{N}\right)\right)$. The concept of exchangeability has, however, the advantage of providing rigorous proofs and thus a deeper characterization of such ensembles. Further, exchangeability has consequences which are much far-reaching than those just discussed. First, the orthonormal bases which have been selected in the present paper are adapted to RME's whose distributions depend on $\operatorname{tr}\left(H_{N}^{\dagger} H_{N}\right)$. For some other RME's, profit can be taken from the freedom in the basis choice to define new random vectors $\mathbf{Y}$, whose components are linear combinations of the previous $\mathbf{X}$ components, so that they are still exchangeable or even $\alpha$ symmetric. In the asymptotic limit, the de Finetti theorem applied to such $\mathbf{Y}$ yields in turn initial RME's as mixtures of RME's whose entries are linear combinations of IID variables. A simple example is a RME $H_{N}$ whose distribution depends on a quadratic form in the elements of $H_{N}$, which reduces to $\mathbf{X}^{T} A \mathbf{X}$ when expressed in terms of $\mathbf{X}$, where $A$ is a symmetric $N_{p} \times N_{p}$ positive definite matrix. Defining $\mathbf{Y}=A^{1 / 2} \mathbf{X}[17]$, the distribution of $\mathbf{Y}$ is spherical and thus the large $N$ distribution of extendible $H_{N}$ is a mixture of RME's whose entries are linear combinations of IID Gaussian variables and may thus be correlated.

Second, the ensembles which are mixed in the de Finetti theorem are not reduced to the sole Gaussian ensembles. For instance, the two-parameter Wigner semicircle is obtained as a limiting distribution for broad conditions on random matrices [2,3] or even on matrices with fixed entries [18]. Every RME, which is asymptotically a mixture of RME's with IID entries whose eigenvalue densities are $\rho_{W}(\lambda)$ [Eq. (1)], has thus a large- $N$ density that is obtained by summing up semicircles with varying square radii $u$ and centering parameters $c$. The weight of a semicircle $(u, c)$ is given by the bivariate distribution of $u$ and $c$.

Third, analogs of de Finetti theorems have also been derived for two-dimensional exchangeable arrays and for stochastic processes [8,19]. Further extensions to realsymmetric RME's, more general than the spherical RME's discussed above, result in fact from a theorem of Kallenberg [19] on infinite random jointly rotatable 2D arrays. Let $H:\left(H_{i j}\right) i, j \geqslant 1$ be such an array and for each $N$, consider the subarray $\left(H_{i j}\right),(1 \leqslant i, j \leqslant N)$ as an $N \times N$ matrix $H_{N} . H$ is jointly rotatable if for each $N, H_{N} \stackrel{d}{=} O_{N} H_{N} O_{N}^{T}$ for all orthogonal $N \times N$ matrices. Kallenberg has proven that an infinite array $H$ is jointly rotatable if and only if it has the following stochastic representation:

$$
H_{i j} \stackrel{d}{=} \omega \delta_{i j}+\tau\left(g_{i j}+g_{j i}\right)+\sum_{k=1}^{\infty} \mu_{k}\left(m_{i k} m_{j k}-\delta_{i j}\right),
$$

where all symbols, except $\delta_{i j}$, denote random variables. Latin symbols stand for IID $N(0,1)$ variables while Greek symbols represent arbitrary random variables independent of the Latin variables. Further, the $\mu_{k}$ 's satisfy $\sum_{k=1}^{\infty} \mu_{k}^{2}<\infty$ [19]. To interpret the various contributions in Eq. (11), we fix first the values of Greek random variables. The $\delta_{i j}$ terms in Eq. (11) are then seen to produce a global shift $c$ of the eigenvalues of the matrix whose elements are given by the sum of the remaining terms. The term $\tau\left(g_{i j}+g_{j i}\right)$ is readily noticed to be an element of a GOE matrix, $N\left[0,2 \tau^{2}(1\right.$ $\left.\left.+\delta_{i j}\right)\right]$. Although different, the matrix associated with the first part of the last sum is reminiscent of random matrices of Marcenko-Pastur ensembles and of some recent extensions of them [20,21]. If the latter matrix belongs to an ergodic RME and if its spectral density is also a Wigner semicircle (see [20,21] for some examples), as it is for the GOE, then the total matrix has a semicircular density, as deduced for instance from Blue's functions [6,7]. However, the latter semicircle is a priori shifted. Letting now the Greek variables vary, we deduce that the asymptotic density of states of the considered ensembles is again given by the sum of shifted Wigner semicircles of varying radii. There are, however, general RME's represented by Eq. (11) whose asymptotic densities do not reduce to such sums. The definition of a criterion to determine beforehand if it is the case or not for a given RME is hence desirable.

The present work yields additionally a simple numerical method of calculation of asymptotic densities in the numerous cases where the latter are built from shifted Wigner semicircles and where closed form formulae cannot be derived. The numerical calculation of $\rho_{\infty}(\lambda)$ may be performed for huge matrices $H_{N}$ as no storage of matrix elements is required to get the characteristics $a$ and $c\left[c=\operatorname{tr}\left(H_{N}\right) / N\right.$ and $\left.a=2\left(\operatorname{tr}\left(H_{N}^{2}\right) / N-c^{2}\right)^{1 / 2}\right]$ of the semicircle associated with a given $H_{N}$. We notice further that properties of distributions of quadratic forms $[17,22]$, here $\operatorname{tr}\left(H_{N}^{2}\right)$, may be helpful to check such numerical results and for theoretical [Eq. (11)] purposes. Similar numerical methods may be applied to matrices with complex eigenvalues [16].

To conclude, the present paper points out the significance of the concept of exchangeability in RMT which reinforces amongs others the importance of the classical Gaussian ensembles. The problems of the extension of the Kallenberg representation to Hermitian matrices and of its explicit construction for RME's which are relevant in physics are finally raised.

G.L.C. thanks the CNRS, NSF, Ministère des Affaires Etrangères, and the Division of Engineering and Applied Science (California Institute of Technology) for financial support, Professor B. Fultz and his group for their welcome, and P. Bogdanoff for a careful reading of the manuscript.
[1] T. Guhr, A. Müller-Groeling, and H. A. Weidenmüller, Phys. Rep. 299, 189 (1998).

[2] M. L. Mehta, Random Matrices, revised and enlarged 2nd ed. (Academic, New York, 1991).

[3] A. M. Khorunzhy, B. A. Khoruzhenko, and L. A. Pastur, J. Math. Phys. 37, 5033 (1996).
[4] P. Neu and R. Speicher, J. Stat. Phys. 80, 1279 (1995).

[5] R. Gopakumar and D. J. Gross, Nucl. Phys. B 451, 379 (1995).

[6] E. Brézin and A. Zee, Nucl. Phys. B 402, 613 (1993); A. Zee, ibid. 474, 726 (1996).

[7] R. A. Janik, M. A. Nowak, G. Papp, and I. Zahed, Acta Phys. Pol. B 28, 2949 (1997). 
[8] D. J. Aldous, Ecole d'Eté de Probabilités de Saint-Flour XIII1983, edited by P. L. Hennequin, Lecture Notes in Mathematics Vol. 1117 (Springer, Berlin, 1985), p. 1.

[9] P. W. Diaconis, M. L. Eaton, and S. L. Lauritzen, Scand. J. Statist. 19, 289 (1992).

[10] K. T. Fang, S. Kotz, and K. W. Ng, Symmetric Multivariate and Related Distributions (Chapman and Hall, London, 1990).

[11] Y. L. Tong, The Multivariate Normal Distribution (Springer, Berlin, 1990).

[12] T. Gneiting, J. Multivariate Anal. 64, 131 (1998).

[13] I. J. Schoenberg, Ann. Math. 39, 811 (1938).

[14] P. Ressel, Ann. Prob. 13, 898 (1985).
[15] P. Cizeau and J. P. Bouchaud, Phys. Rev. E 50, 1810 (1994).

[16] G. Le Caër and R. Delannay, STATPHYS20, Paris, July 2025, 1998 (unpublished).

[17] A. M. Mathai and S. B. Provost, Quadratic Forms in Random Variables (Dekker, New York, 1992).

[18] L. Arnold, Linear Algebr. Appl. 13, 185 (1976).

[19] O. Kallenberg, Prob. Theory Rel. Fields 77, 415 (1988).

[20] V. Marchenko and L. Pastur, Math. USSR Sb. 1, 457 (1967).

[21] A. Khorunzhy and G. J. Rodgers, J. Math. Phys. 38, 3300 (1997).

[22] A. M. Mathai, S. B. Provost, and T. Hayakawa, Bilinear Forms and Zonal Polynomials (Springer, New York, 1995). 